empty cart appears to have gone over him, at Kingston, three miles from Bedford. He was immediately taken to the Bedford Infirmary. On his admission, he was slightly sensible. There was a superficial cut on the back of the bead, and a copious flow of blood from the right ear. On the following day, clear fluid began to escape from the ear, and continued to do so. The same day he was better, and still better on the third. On the fourth day he became sleepy; comatose symptoms set in, and he died early on the fifth day. On post mortem examination, the posterior surface of the petrous bone was found fractured across; there was also a considerable clot of blood (an ounce and a half to two ounces) between the dura mater and the skull on the left side ; also considerable recent inflammation on the under surface of the cerebellum.

CASE IX. - Extensive Fracture of Base of Skull from Violent Pressure on Both Sldes: Deatiz after Trvo Hours.-W. F., an agricultural labourer, aged 34, fell from the shaft of a waggon on which he was sitting driving at Bromham, Bedfordshire, when the waggon-wheel ran over him near the head. When taken up, he was insensible, bleeding from both nostrils, and from the right ear. He died immediately after admission to the Bedford Infirmary, about two hours subsequently to the accident. On post mortem examination, there was found a large quantity of extravasated blood on the surface of the brain, the side of the skull fractured through the squamous portion of the right temporal bone, and the base extensively fractured through the petrous portion.

CASE X.-Extensive Fracture of Base of Skull from Violent Pressure on both Sides: Recovery. - About twenty-five years since, a bricklayer had his head jammed between two buffers of a train (cracked like a cocoa-nut, in fact). Upon his arrival at the Bedford Infirmary, he was quite insensible, with blood flowing from both ears, the nose, and mouth. For several days after the accident, there was a watery discharge from both ears. Consciousness returned in two or three weeks, and he progressed favourably but slowly, and left the Hospital in about five months. During the time he was in the Infirmary, he suffered from severe pains in the head, which continued for a long time after he was discharged, and the nuuscles on the right side of the face became paralysed. $\mathrm{He}$ is at the present time following his usual employment as a bricklayer, and is in good health; but there is still want of power in the muscles of the right side of the face.

CASE XI.-Extensive Fracture of Base of Skull from a Violent Blow, and Lateral Pressure: Death.-T. S., a child aged 5, in the summer of 1867 was watching the men at work at the Midland Ouse Bridge at Bedford. One of the men engaged was carving a balk of timber, when the iron rod broke, and the end of the balk fell on the child's head, crushing it into the ground. Death was almost instantaneous. Blood flowed abundantly from the mouth and ears. On examination, no fracture could be found at the upper part of the skull ; but it was detected at the junction of the occipital with the parietal bone, and probabiy extended across the base of the skull.

The last three cases are examples of the greatest degree of violence which this part can sustain with the faintest possibility of recovery. In each the violence was excessive ; in each pressure operated from both sides; in one, death was almost or quite instantaneous; in another, the patient survived for two hours ; in the third astonishing, and, I should think, almost unique case, the subject is living to this day.

\section{CASE OF CARBOLIC ACID POISONING.}

By ALEXANDER OGSTON, M.D., Assistant-Professor of Medical Jurisprudence in the University of Aberdeen.

ON October 26th, 1870, Patrick McGrath, aged 47, sergeant-instructor of volunteers, Tarves, found in a rifle-shed a bottle of crude carbolic acid, part of which he swallowed, in mistake for bitters or other fluid. It is calculated that he swallowed, at most, an ounce or two of the fluid. This, when examined, seemed to be of considerable strength, crude, black, and oily. He then proceeded to a druggist's shop, but left this at once, without taking any drug. Shortly afterwards, he was found lying insensible, and was taken to Dr. Irvine's house. Dr. Irvine kindly handed me his notes of the case, which are as follows.

"Last night, October 26th, P. McG. was brought to my house at 5.30 P. M. He was reported to have been accidentally poisoned. He had a strong smell of carbolic acid, was unable to speak or walk, and was quite unconscious and in a state of apparent apoplexy, with stertorous breathing and puffing of the lips at each expiration. The pulse was not much disturbed. The heat of surface was natural. I laid him on a couch, covered him warmly, and administered castor-oil to dilute and carry off the poison." The bottle containing the poison was distinctly labelled "Carbolic Acid, Poison." The notes continue: "On coming back, I tried with my patient olive-oil and a powerful emetic, which had no effect. I then sent for my son, who brought a stomach-pump; it would not work, and was consequently not applied. Subsequently, carbonate of ammonia and brandy were tried to no purpose. The patient's lips, gums, and tongue were white, as if sodden. After he had been laid on the couch, there was for three or four hours no muscular movement at all. The eyelids were closed, and the pupils contracted to the size of a pin-point. The first indications of returning sensibility were in the eyelids. About 12 o'clock, he began to open his eyes to some extent. Subsequently, he moved his arms, then his feet and legs ; and afterwards made an attempt to raise his shoulders. After this, the pupils gradually expanded, but never became large. He began to speak, and asked for cold water to drink; but he never acquired complete consciousness. The next phase was affection of the lungs. There was very hard and laborious breathing, and difficult muco-purulent expectoration. After some time, the breathing became easier, just as suddenly as the coma had improved. He then complained of severe pain in the back. The pulse, which at first was not much disturbed, ran up to $84,90,96$, and 106-not always alike, but fluctuating. $\mathrm{Re}$ spiration from 46 to 48 . At 2 o'clock in the morning, he passed naturally a quantity of very dark coloured urine, having the smell of carholic acid. He afterwards attempted again to pass urine, but without success. No real reaction ever took place, notwithstanding the frequent use of warm applications. The brow, face, and hands were covered with cold clammy perspiration. Collapse came on, and at 7 A.M. death closed the scene, after thirteen hours and thirty-five minutes of suffering."

On proceeding to examine the body thirty-two hours after death, it was found in the following state. Rigor mortis was present. There was lividity of the back parts, and of the front of the neck and chest. The face was sallow. The pupils were natural. Watery froth and yellowish fluid, smelling of carbolic acid, were present at the mouth and nose. There was a clot in the superior longitudinal sinus, The dura mater and arachnoid were congested, the blood smelling of carbolic acid. Serum, with a distinct odour of carbolic acid, was found in the subarachnoid tissue and in the ventricles. The cineritious brainmatter was pinkish in hue. There was intense congestion of the vessels on the under surface of the pons Varolii and medulla oblongata. The mucous membrane of the mouth, throat, and gullet was unnaturally white and sodden in appearance, and at parts was soft and easily detached. There were two fluid-ounces of thick brownish pulp, smelling of carbolic acid, in the stomach. At its greater curvature, the mucous membrane of the stomach was studded with hard, reddish, and elevated points, of the size of small shot, without any marked congesgestion around them. Similar but smaller points existed on other parts of the stomach-showing, however, a more linear form, and tending to follow the course of the vessels. The stomach was otherwise natural, or perhaps a little more vascular than usual. Portions of the small intestine were slate-coloured, especially in the duodenum and upper part of the jejunum, but otherwise natural in every way, and containing a brownish pulpy matter. The salivary glands, pancreas, and mesenteric glands, were all pale. The heart was healthy, and full of dark firmly clotted blood on both sides; the right side containing four, and the left side two ounces. A minute fibrinous clot was found in the pulmonary artery, imbedded in a large black clot. The trachea was congested, and contained fluid similar to that found at the mouth and nostrils. The lungs were bulky and very œdematous. Fatty patches were present on the upper surface of the liver. The gallbladder was enormously distended with bile. The spleen was soft; the kidneys fatty. Four ounces of turbid urine were found in the bladder. The blood, urine, and solid textures of the body, all smelt of carbolic acid. The blood was every where very firmly clotted.

\section{ON SYPHILITIC RENAL DROPSY.}

By S. MESSENGER BRADLEY, F.R.C.S., Lecturer on Human and Comparative Anatomy, Royal School of Medicine and Surgery, Manchester.

MEDICAL reasoning is too often hastily deductive in character ; in other words, we too often reason from the particular to the universal, and rush from an unproven hypothesis to an erroneous and dogmatic conclusion. This constitutes the very sin of the Aristotelian method, and, if followed constantly, would bring medical science into equally welldeserved disrepute, as it did the old Grecian philosophy.

Still, reasoning thinking men will draw deductions; nay, more, we 